Research Paper

20184121

\title{
Trajectory Prediction of Surrounding Vehicles Considering Individual Driving Characteristics
}

\author{
Hanwool Woo ${ }^{1)}$ Yonghoon Ji ${ }^{1)} \quad$ Yusuke Tamura $^{1)}$ Yasuhide Kuroda $^{2)}$ Takashi Sugano $^{2)}$ Yasunori Yamamoto $^{2)}$ \\ Atsushi Yamashita 1) Hajime Asama 1) \\ 1) Department of Precision Engineering, The University of Tokyo \\ 7-3-1 Hongo, Bunkyo-ku, Tokyo 113-8656, Japan (E-mail: woo@robot.t.u-tokyo.ac.jp) \\ 2) Mazda Motor Corporation \\ 2-5 Moriyacho, Kanagawa-ku, Yokohama 221-0022, Japan \\ 3-1 Shinchi, Akigun Fuchucho, Hiroshima 730-8670, Japan
}

Received on July 24, 2018

\begin{abstract}
We propose a method to predict trajectories of surrounding vehicles considering individual driving characteristics. Trajectory prediction of surrounding vehicles is attracting a lot of attention now, and it is expected to apply to advanced driver assistance systems. However, previous methods perform the trajectory prediction based on common driving patterns even though each driver shows a different driving characteristic. The proposed method focuses on the following behavior behind the preceding vehicle and estimates a driving characteristic of each driver using machine learning techniques. Based on the estimation result, the proposed method adjusts the prediction model and appropriately generates a trajectory. As the result, the performance of trajectory prediction can be dramatically improved.
\end{abstract}

KEY WORDS: Safety, Accident avoidance/Collision prediction, Intelligent/Computer application [C1]

\section{Introduction}

According to a survey by the Japan Metropolitan Police Department, over $90 \%$ of car crashes are caused by human mistakes ${ }^{(1)}$. Recently, autonomous driving technologies and advanced driver assistance systems have attracted considerable attention as solutions for preventing car crashes. The implementation of intelligent technologies to assist drivers in recognizing situations around their own vehicles can be expected to decrease the accident rates. Car crashes often occur when traffic participants attempt to change lanes as shown in Fig. 1. If the safety support system of egovehicle can predict lane-changing trajectories of surrounding vehicles; accident rates can be significantly decreased.

There are many previous studies about the trajectory prediction. Wolf and Burdick proposed a method for an autonomous vehicle by applying the potential field method ${ }^{(2)}$. However, this method can only be used to calculate self-trajectory, as the desired velocity needs to be known in order to calculate the potential energy. Therefore, their method is not suitable for the trajectory prediction of surrounding vehicles. Houenou et al. proposed a method based on a motion model and a maneuver recognition model ${ }^{(3)}$. Kasper et al. also used a method to predict a lane-changing maneuver by the regression analysis ${ }^{(4)}$. However, these methods do not consider adjacent vehicles when they generate a trajectory. Furthermore, previous methods perform the prediction based on common driving patterns even

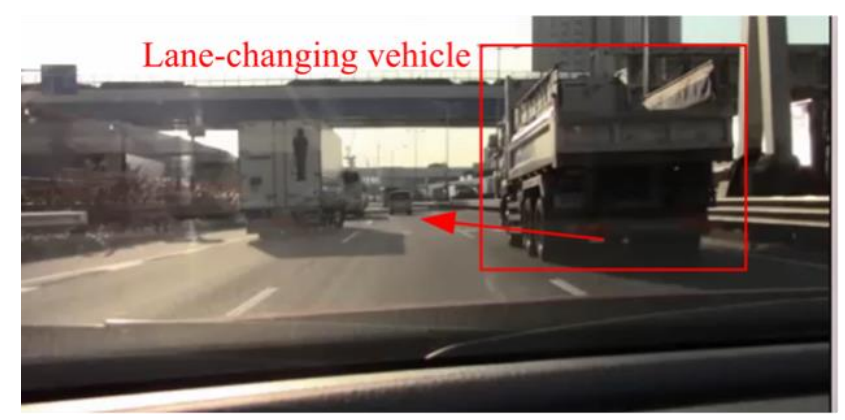

Fig. 1 Lane change of surrounding vehicle: the target of trajectory prediction is a surrounding vehicle driving near the ego vehicle containing the measurement devices. The proposed method predicts the trajectory of the lane-changing vehicle indicated by the red rectangle in the figure.

though each driver shows a different driving characteristic. Generally, drivers have different driving characteristics influenced by several conditions such as personality, driving environment, and experiences ${ }^{(5)(6)}$. According to a previous research ${ }^{(7)}$, driving characteristics were found to affect driving patterns in unique ways even if the drivers were under similar conditions, thus limiting the performance of prediction systems.

In order to solve this issue, it is necessary to determine driving characteristics of surrounding drivers and adjust the trajectory prediction model according to their characteristics. Quintero et al. employed an approach to classifying a driver as exhibiting either an aggressive or moderate driving characteristic ${ }^{(8)}$. This method 
analyzes the changes in the longitudinal and lateral positions by using the throttle, brake, and steering as features. Aljaafreh et al. defined driving characteristics and categorized them into below normal, normal, aggressive, and very aggressive ${ }^{(9)}$. They proposed a method to estimate the characteristic based on acceleration patterns. The acceleration and deceleration in the longitudinal and lateral directions were used as features. However, these features cannot be measured from within the ego vehicle. Therefore, it is impossible to estimate the driving characteristics of surrounding drivers using these factors.

To overcome the above limitations, we propose a novel method to predict trajectories of surrounding vehicles considering individual driving characteristics. Most of previous methods need to determine values of parameters in the trajectory prediction, and they use machine learning techniques so that the performance is maximized. However, this approach has a problem that individual differences cannot be considered. To solve this problem, the proposed method estimates the driving characteristic of the target driver and adjusts parameters of the trajectory prediction method. Our previous work was proposed to appropriately determine the driving characteristic of the target vehicle which follows the preceding vehicle ${ }^{(10)}$. Only measurable information regarding the ego vehicle is used, thus, it is possible to estimate the driving characteristics of surrounding drivers. The proposed method uses a potential field method toward to the longitudinal direction and a sinusoidal model in the lateral direction. The potential field method is generally used for path planning in robotics ${ }^{(11)(12)}$. This method sets a goal to generate an attractive potential energy and obstacles to generating a repulsive potential energy. By setting the environment, it can generate both paths to keep the current lane or change a lane ${ }^{(13)}$. However, parameters have constant values in previous methods, as the result, the performance of trajectory prediction may degrade depending on conditions. The proposed method adjusts the values of parameters based on the result of driving characteristic estimation to improve the prediction performance.

The remainder of this paper is organized as follows. Section 2 presents the problem definition and the overview of the proposed method. Section 3 describes the trajectory prediction method. Section 4 explains the experiments using a driving simulator and presents the evaluation results. Finally, Section 5 presents the conclusions and future work.

\section{Overview}

\subsection{Problem definition}

In this study, the scene is modified to obtain a straight two-lane infinite highway, which has only one side as shown in Fig. 2. The ego

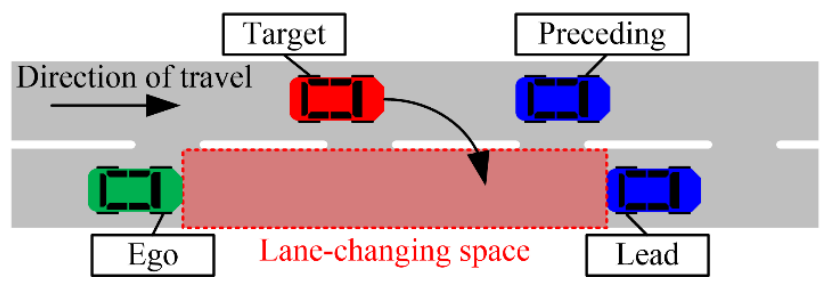

Fig. 2 Problem setting: the proposed method focuses on a situation when the target vehicle cuts in the front space of the ego vehicle since it is the main factor of accidents.

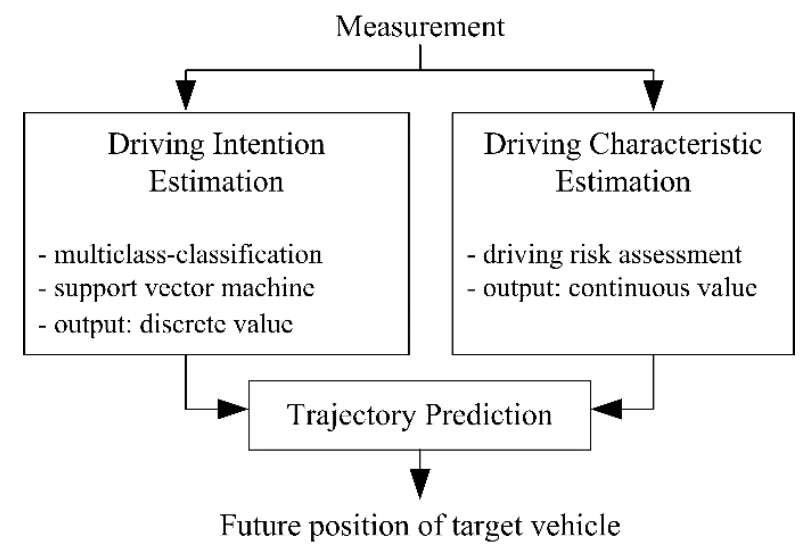

Fig. 3 Overview of proposed method

vehicle, indicated using green color, houses measurement devices such as a GPS and laser scanners and predicts a trajectory of the target vehicle which is the red one. The ego vehicle also estimates the driving characteristic of the target vehicle. The proposed method focuses on a situation wherein the target follows the preceding vehicle, the driving characteristic is estimated at each time step. The revision to previous studies shows a prevalence of driving characteristic estimation using either two or three levels. One of the motivation to estimate the driving characteristic is to detect aggressive drivers in surrounding vehicles. As these drivers show a risky driving pattern (e.g., improper position, inconsistent or excessive acceleration), it is strongly required to react to their behavior as soon as possible. In this study, the driving characteristic is categorized into two levels: moderate and aggressive, as observed in a previous study ${ }^{(7)}$. The driving characteristic of the target is derived.

Based on the estimated characteristic, a lane-changing trajectory of the target is predicted until two seconds in the future. The proposed method focuses on a situation when the target vehicle cuts in the front space of the ego vehicle since it is the main factor of accidents. Therefore, the proposed method is mainly designed to predict the lane-changing trajectory, of course, it can be used to the lane-keeping situation. The construction and evaluation of the proposed method are conducted with respect to lane-changing situations.

\subsection{Overview of proposed method}


Class: 1

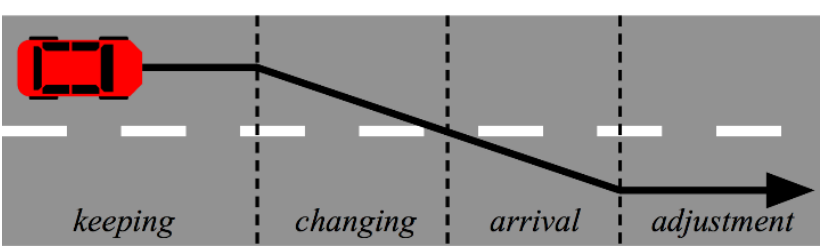

Fig. 4 Driving intentions: the proposed method defines that drivers have four intentions when they change lanes: keeping, changing, arrival, and adjustment.

To overcome the limitation of the previous methods, the proposed method adjusts the trajectory prediction model based on the individual driving characteristics. Figure 3 shows the schematic of the proposed method. It comprises the three parts: driving characteristic estimation, driving intention estimation, and trajectory prediction. Inputs of the method are the positions of the ego vehicle and surrounding vehicles. The position of the ego vehicle can be measured by a GPS and that of surrounding vehicles can be acquired by laser scanners. Using this information, the features are extracted for the driving characteristic estimation and the driving intention estimation.

First, the driving characteristic estimation is performed using three information: relative velocity, relative distance, and repulsive potential energy. These relative numbers are calculated between the target vehicle and the preceding vehicle. The proposed method considers the driving risk, which is defined as the possibility of colliding with the preceding vehicle. The repulsive potential energy is used to evaluate the driving risk by using the dynamic potential field method. Details of this method are explained in our previous paper ${ }^{(10)}$.

Second, the proposed method defines that drivers have four intentions when they change lanes: keeping, changing, arrival, and adjustment as shown in Fig. 4. Each driving intention is defined as a class, and the proposed method treats the driving intention estimation as a multiclass problem by the SVM (support vector machine). The method is based on the lateral movement of the target vehicle. Details of this method are explained in our previous papers ${ }^{(13)(14)}$. The output of this part is the intention at the current time.

Third, the results of above two parts are used for the trajectory prediction. The proposed method uses the potential field method, and the estimated driving characteristic of the target vehicle is used to adjust the parameters. The values of parameters determine acceleration and deceleration tendency of the driver, and they largely affect the performance. The trajectory prediction adopts the result of driving intention estimation to identify the strategies that drivers may execute while driving. Generally, drivers execute different strategies with different driving intentions. When drivers have intentions such as keeping and adjustment, they aim at the front of the current lane and pay more attention to the vehicle in the same lane than vehicles in the other lanes. They may attempt to remain at the center of the lane.

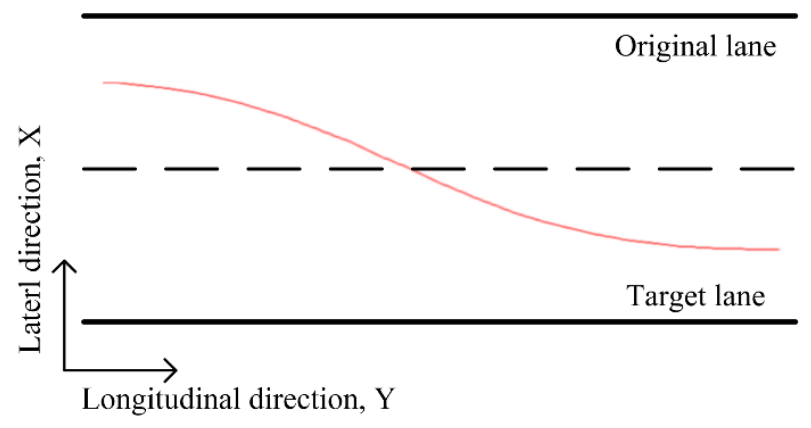

Fig. 5 Sinusoidal model for lane-changing trajectory.

On the other hand, when drivers have intentions such as changing and arrival, they aim at the front of the next lane and must consider adjacent vehicles on not only the current lane but also the next lane. Therefore, in addition to the strategy, drivers must consider surrounding vehicles while driving. The proposed method changes the strategy according to the estimated intention. The detail of this method is discussed specifically in Section 3.

\section{Trajectory prediction}

The proposed method predicts a trajectory according to the estimated driving intention of the target driver. First, when the driving intention is keeping and adjustment, the goal is set to the front of the current lane. The proposed method sets the goal to the center of the current lane. The surrounding vehicles generate the repulsive potential energy, and it makes the target keep a gap between front and back. However, the driver is not concerned with the vehicles in the next lane.

On the other hand, when the driving intention is changing or arrival, the driver aims at the front of the next lane and may check the gap with vehicles in both lanes. The proposed method uses the sinusoidal model to generate a lane-changing trajectory in the lateral direction and the potential field method to avoid surrounding vehicles in the longitudinal direction. Details are explained as follows.

\subsection{Prediction of lateral movement}

For the prediction of lateral movement in lane-changing, the proposed method uses the sinusoidal model ${ }^{(15)}$. This model is able to generate a lane-changing trajectory as shown in Fig. 5, and it does not require a parameter. The acceleration in the lateral direction can be derived as

$$
a_{\text {lat }}(t)=\frac{2 \pi H}{t_{\text {lat }}^{2}} \sin \frac{2 \pi}{t_{\text {lat }}} t,
$$


where $a_{\text {lat }}$ represents the lateral acceleration, $t$ is the time from the beginning of lane-changing, $H$ is the final lateral displacement, and $t_{\text {lat }}$ is the lane-changing duration. The proposed method determines the value of $H$ as the lane width. Furthermore, $t_{\text {lat }}$ is calculated using the lateral velocity at the moment when the intention is estimated as changing. Thus, the lateral acceleration can be calculated without any parameters.

\subsection{Prediction of longitudinal movement}

The proposed method predicts the longitudinal movement by using the potential field method. It defines two potential energies and generates a trajectory of the target vehicle to a goal while avoiding adjacent vehicles. The total potential energy at the position $(x, y)$ is derived as

$$
U(x, y)=U_{g}+U_{s}
$$

where $U_{g}$ denotes the attractive potential energy from the goal, and $U_{S}$ denotes the repulsive potential energy from surrounding vehicles. Moreover, $x$ denotes the lateral position of the target vehicle, and $y$ is the longitudinal position of that as shown in Fig. 5. First, the potential energy from the goal is calculated as

$$
U_{g}(y)=-\omega_{g} y,
$$

where $\omega_{g}$ is the weight coefficient. The value of $\omega_{g}$ determines acceleration/deceleration tendency when there is no surrounding vehicle. The large value makes sudden velocity changes, and it makes a close vehicle gap between the target vehicle and the preceding vehicle. This tendency is normally considered as the behavior of aggressive drivers. This is one of the parameters which should be adjusted according to the driving characteristic.

On the other hand, the repulsive potential energy from surrounding vehicles is calculated as

$$
U_{s}(y)=-\omega_{s} \exp \frac{\left(y-y_{p}\right)^{2}}{\sigma^{2}}
$$

where $\omega_{s}$ is the weight coefficient, $\sigma$ is the standard deviation of a gap between the target and preceding vehicle, and $y_{p}$ is the position of the preceding vehicle. The values of $\omega_{s}$ and $\sigma$ determine how the target vehicle takes a gap from the preceding vehicle. The large value of $\omega_{s}$ makes enough gap between the two vehicles. $\sigma$ is determined referenced by the previous study ${ }^{(16)}$, and $\omega_{s}$ is the parameter which should be adjusted by the driving characteristic.

As explained previously, the proposed method adjusts above two parameters according to the estimated driving characteristic. Our previous work used the SVM and performed a binary classification ${ }^{(10)}$. On the other hand, the proposed method defines the repulsive potential energy from the preceding vehicle as the driving characteristic variable and uses it for the adjustment of the above two parameters. By this approach, a computation cost is significantly shortened since the classification by the SVM, which requires a high computation, is not performed. The driving characteristic variable $\eta$ can be derived as

$$
\begin{gathered}
G\left(\Delta V_{p}\right)=\frac{1}{2 \pi I_{0}\left(k\left(\Delta V_{p}\right)\right)} \exp \left(-k\left(\Delta V_{p}\right)\right), \\
H\left(D_{p}\right)=\frac{1}{2 \pi \sigma} \exp \left(-\frac{D_{p}^{2}}{2 \sigma^{2}}\right), \\
\eta=\varepsilon G\left(\Delta V_{p}\right) H\left(D_{p}\right),
\end{gathered}
$$

where $\Delta V_{p}$ represents the relative velocity between the target and the preceding vehicle, $D_{p}$ is the vehicle gap between the two vehicles, $\sigma$ is the standard deviation of the vehicle gap, and $\varepsilon$ is a coefficient. Equation (5) represents the von Mises distribution, and $I_{0}(\cdot)$ is the modified Bessel function of order zero. If the target driver exhibits a moderate driving characteristic, the driver would maintain a considerable distance from the preceding vehicle and drive safely. However, when the target driver has an aggressive driving characteristic, the driver closely follows the preceding vehicle with a high risk of crashing. Moreover, the driver would rapidly approach to surrounding vehicles and change a lane while accelerating. The driving characteristic variable can describe these tendencies by the value of potential energy. A large value represents an aggressive driving characteristic.

The parameters, $\omega_{g}$ and $\omega_{s}$, are adjusted according to the driving characteristic variable, $\eta$. The values are calculated as

$$
\begin{gathered}
\omega_{g}=\alpha_{g} \eta+\beta_{g}, \\
\omega_{s}=\alpha_{s} \eta+\beta_{s},
\end{gathered}
$$

where $\alpha_{g}, \beta_{g}, \alpha_{s}$, and $\beta_{s}$ are coefficients. Both parameters have large values when the driving characteristic variable is large.

\section{Result}

4.1 Experiments

In this study, a driving simulator (DS) named as "D3 Sim 


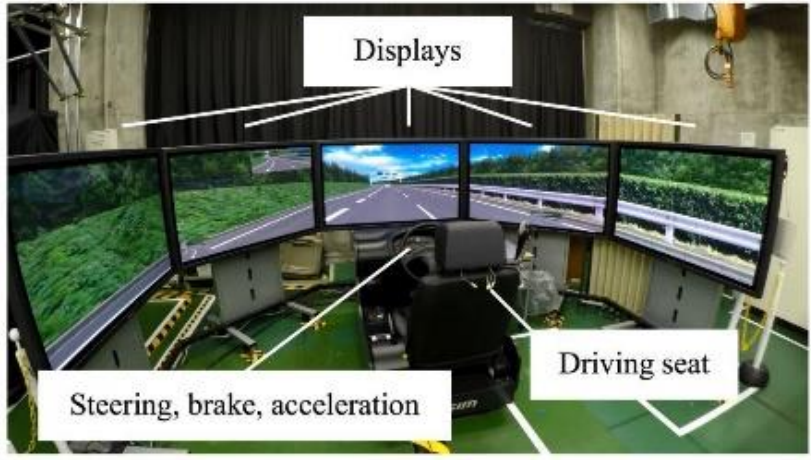

Fig. 6 Driving simulator: D3 Sim was used to acquire data for the evaluation. Display devices consist of five monitors, and a driving seat is located to the center of monitors. The driving simulator includes a steering wheel, an acceleration pedal, and a braking pedal. In addition, audio devices are also installed to make realistic environment.

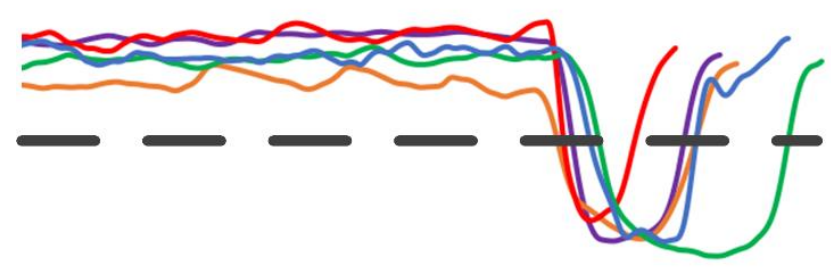

Fig. 7 Comparison of driving trajectories of subjects: the subjects performed to overtake the preceding vehicle, then, returned to the original lane. Colors depict each subject's driving pattern at the first trial. It can be confirmed that there are differences of driving pattern according to their own driving characteristic.

(Mitsubishi Precision Co., LTD.)" was used to collect the data to develop the estimation model and the testing data for evaluation. This simulator showed visual information on display devices comprising five monitors as shown in Fig. 6. In addition, the driving seat comprises a steering, an acceleration pedal, and a brake pedal. The data were recorded at $120 \mathrm{~Hz}$. A total of ten subjects of different ages participated in the experiment with different simulator/driving experiences. Their informed consent was obtained from all the subjects. This paper was approved by the Institute Review Board of Mazda Motor Corporation.

An experimental scene was modified wherein a straight two-lane infinite highway having only one side was considered. The red car is the vehicle operated by the subjects in the experiments, the blue one is the preceding vehicle. Moreover, there is the lead vehicle, which is in a lane adjacent to that of the target vehicle. Both the preceding and lead vehicles blocked the roads of the subject vehicle while the velocities changed randomly. Thus, the subject vehicle was not allowed to overtake them. Consequently, the target was forced to follow the preceding vehicle for $60 \mathrm{~s}$ per one trial. Twenty trials were

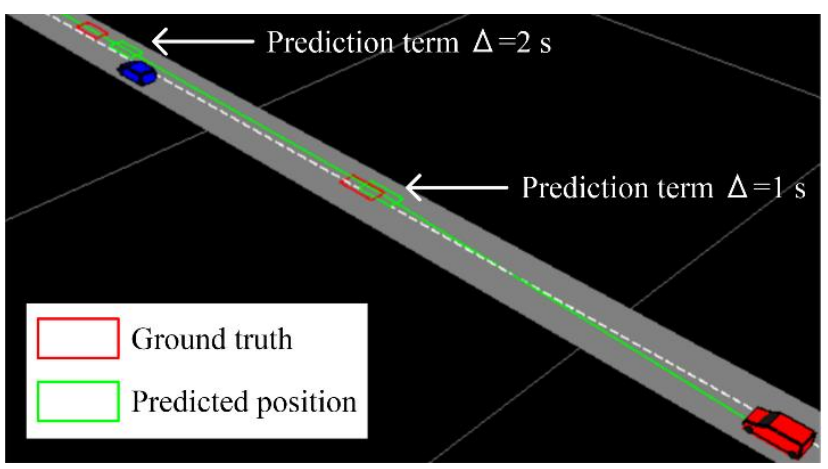

Fig. 8 Three prediction terms: the performance evaluation was performed at two prediction terms: $1 \mathrm{~s}$ and $2 \mathrm{~s}$ later. The red rectangle represents the ground truth at the terms, and the green rectangles are the predicted positions by the proposed method.

Table 1 Performance of proposed method.

\begin{tabular}{ccccc}
\hline \hline & \multicolumn{2}{c}{ Average } & \multicolumn{2}{c}{ Standard deviation } \\
\hline $\begin{array}{c}\text { Prediction } \\
\text { term } \Delta\end{array}$ & $\begin{array}{c}\text { Lateral } \\
\text { error }\end{array}$ & $\begin{array}{c}\text { Longitudinal } \\
\text { error }\end{array}$ & $\begin{array}{c}\text { Lateral } \\
\text { error }\end{array}$ & $\begin{array}{c}\text { Longitudinal } \\
\text { error }\end{array}$ \\
\hline $1 \mathrm{~s}$ & $0.36 \mathrm{~m}$ & $0.44 \mathrm{~m}$ & $0.08 \mathrm{~m}$ & $0.29 \mathrm{~m}$ \\
$2 \mathrm{~s}$ & $0.40 \mathrm{~m}$ & $1.73 \mathrm{~m}$ & $0.18 \mathrm{~m}$ & $1.17 \mathrm{~m}$ \\
\hline \hline
\end{tabular}

conducted per a subject. To evaluate an inherent nature of each subject, the data was measured without any instruction about the driving characteristic as the result, it should be expected to record driving patterns according to own individual characteristic. Totally, 200 trials were conducted in the experiments.

Figure 7 shows driving patterns at the first trial among twenty trials of five subjects. Colors depict each subject's driving pattern. The subjects performed to overtake the preceding vehicle, then, returned to the original lane. It is clearly shown that there are differences in driving patterns by the subjects. This result shows that driving patterns should be different according to the individual driving characteristics, as the result, it should be considered for the performance of trajectory prediction.

\subsection{Performance evaluation}

The trajectory prediction is performed until two seconds in the future. In this study, the prediction performance was evaluated at two prediction terms: $1 \mathrm{~s}$ and $2 \mathrm{~s}$ later, as shown in Fig. 8 . The red rectangle represents the ground truth at each prediction term, and the green rectangle denotes the predicted position by the proposed method. The accuracy can be evaluated by the error between the ground truth and the predicted position.

Table 1 shows the evaluation result by the proposed method. First, it is confirmed that the accuracy for the case of short-term was obviously more precise than that of long-term prediction. Second, the 
Table 2 Performance comparison.

\begin{tabular}{c|ccc}
\hline \hline Method & Prediction term $\Delta$ & Lateral error & Longitudinal error \\
\hline $\begin{array}{c}\text { Dynamics } \\
\text { Proposed method } \\
\text { (w/o characteristics) } \\
\text { Proposed method }\end{array}$ & $2 \mathrm{~s}$ & $1.02 \mathrm{~m}$ & $7.32 \mathrm{~m}$ \\
\hline \hline
\end{tabular}

prediction in the lateral direction was better than the longitudinal movement since the displacement in the lateral direction was smaller during lane-changing. Note that for $\Delta=2 \mathrm{~s}$, the error in the lateral direction was under $0.5 \mathrm{~m}$, and it is the better accuracy compared to that of the previous method even though the different dataset was used for evaluation ${ }^{(17)}$. The requirements for collision avoidance is to identify whether the target vehicle is in the path of the ego vehicle. Therefore, the criterion can be defined that the lateral error is within $1.5 \mathrm{~m}$ according to the previous research ${ }^{(18)}$. Based on statistics, all values lie within three standard deviations of the mean with $99.7 \%$ probability as near certainty. The lateral error of the proposed method at the time horizon $2 \mathrm{~s}$ is $0.94 \mathrm{~m}$ three standard deviations of the mean as shown in Table 1. As the result, it can be concluded that the performance of proposed method satisfies the criterion.

In order to confirm the validity considering the driving characteristic, the performance was compared to the dynamic model and the method without the adaptation of characteristics. Table 2 shows the result of performance comparison. It is clearly confirmed that the proposed method with the adaptation of driving characteristics is able to significantly improve the prediction accuracy. Compared to the error of dynamics, that of the proposed method was dramatically decreased, especially the longitudinal error. Moreover, the accuracy in the longitudinal direction was also improved by considering the driving characteristics. To confirm whether there are significant differences by the adaptation of driving characteristics, the Wilcoxon signed-ranks test was conducted. The sample size was the number of subjects, thus, the test statistic value was compared to the critical value at 0.05 level. By this test, it was confirmed that there are significant differences between the proposed method and the method without the adaptation of driving characteristics in the prediction accuracy. From above results, it was demonstrated that the proposed method achieved the great performance in the trajectory prediction.

\section{Conclusion}

In this study, we proposed a novel method to predict trajectories of surrounding vehicles considering individual driving characteristics. Previous methods have the problem that performs the prediction based on common driving patterns even though each driver shows the different driving characteristic. Through comparison with previous approaches, it was confirmed that the proposed method with the adaptation of the individual characteristics can improve the accuracy of trajectory prediction. The reason for the improvement is expected to be due to the fact that acceleration/deceleration in the longitudinal direction is largely influenced by the driving characteristics. Aggressive drivers normally show inconsistent or excessive acceleration/deceleration, and that tendency should be considered to properly predict their behavior. The proposed method adapts the individual differences, while previous methods do not.

As future work, the individual differences should be considered toward not only the longitudinal direction but also the lateral direction. In this study, the proposed method uses the sinusoidal model for the lateral movement. We have continuously evaluated other approaches that are expected to improve the performance.

This paper is written based on a proceeding presented at JSAE 2018 Annual Congress.

\section{References}

(1) Japan Metropolitan Police Department: States of occurrence of traffic accidents (2014).

(2) M. T. Wolf and J. W. Burdick: Artificial potential functions for highway driving with collision avoidance, Proceedings of the IEEE International Conference on Robotics and Automation, pp. 37313736 (2008)

(3) A. Houenou, P. Bonnifait, V. Cherfaoui, and W. Yao: Vehicle trajectory prediction based on motion model and maneuver recognition, Proceedings of the 2013 IEEE/RSJ International Conference on Intelligent Robots and Systems, pp. 4363-4369 (2013). (4) D. Kasper, G. Weidl, T. Dang, G. Breuel, A. Tamke, and A. Wedel: Object-oriented bayesian networks for detection of lane change maneuvers, Proceedings of the 2011 IEEE International Conference on Intelligent Vehicle Symposium, pp. 673-678 (2011).

(5) B. Shi, L. Xu, J. Hu, Y. Tang, H. Jiang, W. Meng, and H. Liu: Evaluating driving styles by normalizing driving behavior based on personalized driver modeling, IEEE Transactions on Systems, Man, and Cybernetics: Systems, Vol. 45, No. 12, pp. 1502-1508 (2015). 
(6) P. Brombacher, J. Masino, M. Frey, and F. Gauterin: Driving event detection and driving style classification using artificial neural networks, Proceedings of the 2017 IEEE International Conference on Industrial Technology, pp. 997-1002 (2017).

(7) A. Doshi and M. M. Trivedi: Examining the impact of driving style on the predictability and responsiveness of the driver, Proceedings of the 2010 IEEE Intelligent Vehicles Symposium, pp. 232-237 (2010). (8) C. G. Quintero, J. O. Lopez, and A. C. C. Pinilla: Driver behavior classification model based on an intelligent driving diagnosis system, Proceedings of the 2010 IEEE International Conference on Intelligent Transportation Systems, pp. $894-899$ (2010).

(9) A. Aljaafreh, N. Alshabatat, and M. S. N. Al-Din, Driving style recognition using fuzzy logic, Proceedings of the 2012 IEEE International Conference on Vehicular Electronics and Safety, pp. $460-463$ (2012).

(10) H. Woo, Y. Ji, Y. Tamura, Y. Kuroda, T. Sugano, Y. Yamamoto, A. Yamashita, and H. Asama: Dynamic State Estimation of Driving Style Based on Driving Risk Feature, International Journal of Automotive Engineering, Vol. 9, No. 1, pp. 31-38 (2018).

(11) H. Chiang, N. Malone, K. Lesser, M. Oishi, and L. Tapia: Pathguided artificial potential fields with stochastic reachable sets for motion planning in highly dynamic environments, Proceedings of the IEEE International Conference on Robotics and Automation, pp. 2347-2354 (2015).

(12) V. A. M. Jorge, R. Maffei, G. S. Franco, J. Daltrozo, M. Giambastiani, M. Kolberg, and E. Prestes: Ouroboros: using potential field in unexplored regions to close loops, Proceedings of the IEEE International Conference on Robotics and Automation,pp. 2125-2131 (2015).

(13) H. Woo, Y. Ji, H. Kono, Y. Tamura, Y. Kuroda, T. Sugano, Y. Yamamoto, A. Yamashita, and H. Asama: Lane-change detection based on vehicle-trajectory prediction, IEEE Robotics and Automation Letters, Vol. 2, No. 2, pp. 1109-1116 (2017).

(14) H. Woo, Y. Ji, H. Kono, Y. Tamura, Y. Kuroda, T. Sugano, Y. Yamamoto, A. Yamashita, and H. Asama: Automatic detection method of lane-changing intentions based on relationship with adjacent vehicles using artificial potential fields, International Journal of Automotive Engineering, Vol. 7, No. 4, pp. 127-134 (2016).

(15) V. A. Butakov and P. Ioannou: Personalized driver/vehicle lane change models for ADAS, IEEE Transactions on Vehicular Technology, Vol. 64, No. 10, pp. 4422-4431 (2014).

(16) T. Toledo and D. Zohar: Modeling duration of lane changes, Transportation Research Record: Journal of the Transportation Research Board, Vol. 1999, pp. $71-78$ (2007).

(17) B. Kim, C. M. Kang, S. H. Lee, H. Chae, J. Kim, C. C. Chung and J. W. Choi: Probabilistic vehicle trajectory prediction over occupancy grid map via recurrent neural network, arXiv preprint arXiv:1704.07049 (2017).

(18) S. E. Shladover and S. Tan: Analysis of vehicle positioning accuracy requirements for communication-based cooperative collision warning, Journal of Intelligent Transportation Systems, Vol. 10, No. 3, pp. 131-140 (2006). 